\title{
Pulsed-dose-rate post-operative vaginal cuff brachytherapy for endometrial cancer: preliminary report
}

\author{
Krystyna Serkies, MD, PhD, Ewa Pawłowska, MD, Joanna Kaminska, PhD, Joanna Lipniewicz, MSc, \\ Magdalena Narkowicz, MSc, Prof. Jacek Jassem, MD, PhD \\ Department of Oncology and Radiotherapy, Medical University of Gdansk, Poland
}

\begin{abstract}
Purpose: To present our early experience with the post-operative intracavitary pulsed-dose-rate vaginal brachytherapy (PDR VBT) in high-intermediate risk endometrial cancer patients as defined in the PORTEC-2 trial.

Material and methods: The study group included 60 high-intermediate risk endometrial cancer patients with a median age of 64 (range: 57-87) treated between April 2010 and December 2013 with PDR VBT at a dose of 18 Gy (range: 17-27); 1 Gy/pulse, pulses repeated every hour. Prior surgery consisted of a total abdominal hysterectomy and bilateral salpingo-oophorectomy with or without a lymphadenectomy.

Results: After a median follow-up of 23 months there were two local recurrences, both located in the lower part of the vagina, outside of the irradiated area. One of the relapsed women was susccessfully salvaged with surgery followed by pelvic irradiation and remained free of the disease for the subsequent three years. Following therapy, $24(40 \%)$ of the patients experienced grade 1 or 2 urinary and/or bowel toxicity. There was one case with a serious post-surgical (grade 3) urinal side effect.

Conclusions: Post-operative PDR VBT at a dose of $18 \mathrm{~Gy} / 18$ pulses/every hour seems to be a safe technique and ensures vaginal control in high-intermediate risk endometrial cancer patients. Additional follow-up including larger number of patients is necessary for the final assessment of this therapy.

Key words: endometrial carcinoma, brachytherapy, pulsed-dose-rate.

\section{Purpose}

Post-operative vaginal brachytherapy (VBT) is considered an adequate management to prevent vaginal failure in patients with early endometrial cancer [1]. Post Operative Radiation Therapy in Endometrial Carcinoma (PORTEC-2) randomised trial demonstrated similar local control with a better quality of life with VBT, as compared to external beam pelvic irradiation for highintermediate risk endometrial cancer patients [2,3]. In this trial, a dose equivalent to 45-50 Gy was delivered to the vaginal mucosa in the VBT group: $21 \mathrm{~Gy}$ in three fractions of 7 Gy, 1 week apart for high-dose-rate (HDR; 85.4\% of patients); $30 \mathrm{~Gy}$ at $0.5-0.7 \mathrm{~Gy} / \mathrm{h}$ for low-dose-rate (LDR; $8.9 \%$ of patients), and $28 \mathrm{~Gy}$ at $1.0 \mathrm{~Gy} / \mathrm{h}$ in one session for the medium-dose-rate (3.8\% of patients). Here we present our results with 18 Gy pulsed-dose-rate (PDR) VBT in a similar group of endometrial cancer patients.

\begin{abstract}
Material and methods
Between April 2010 and December 2013, 60 consecutive endometrial cancer patients underwent the postoperative PDR VBT procedure at the Medical University of Gdańsk (Table 1). All patients met the inclusion criteria used in the PORTEC-2: 1) over 60 years old and FIGO 1988 stage IC grade 1 or 2 disease, or stage 1B grade 3 disease, and 2) had any age and stage IIA disease, and any grade (apart from grade 3 with greater than $50 \%$ myometrial invasion). The planned dose of $18 \mathrm{~Gy} /$ 18 pulses/every hour specified at $0.5 \mathrm{~cm}$ distance from the external surface of the single-channel cylinder, with the reference isodose covering the proximal $3 \mathrm{~cm}$ of the vagina was administered, except for the first 11 and other 3 patients, who were prescribed a dose of 20-27 Gy and $17 \mathrm{~Gy}$, respectively. Fifty patients had 2D orthogonal filmbased plans, and the remaining 10 had 3D BT. The dosim-
\end{abstract}

\footnotetext{
Address for correspondence: Krystyna Serkies, MD, PhD, Department of Oncology and Radiotherapy, Medical University of Gdańsk, 7 Dębinki St., 80-211 Gdańsk, Poland, phone: +48 5834922 70,

e-mail: kserkies@gumed.edu.pl 
etry was calculated using PLATO, v. 14.1 and Oncentra Master Plan v. 3.1,2 or 3 planning systems (Nucletron, an Elekta company, Elekta AB, Stockholm, Sweden) with or without optimization type: dose points on distance, with manual modifications in some cases. The median interval between surgery and VBT was 74 days (range: 47-116). Treatment plans were assessed retrospectively and the dose at the tip of the applicator for each BT application was extracted. The equivalent dose in 2 Gy fractions (EQD2) at this point for the tumor $(\alpha / \beta=10)$ was calcu-

Table 1. Patient and treatment characteristics $(n=60)$

\begin{tabular}{lc} 
Variable & $n(\%)$ \\
\hline \begin{tabular}{l} 
Age (years) \\
\hline Range
\end{tabular} & $57-87$ \\
\hline Median & 64 \\
\hline FIGO stage (according to FIGO 1988 staging) & \\
\hline IC & $41(68.3)$ \\
\hline IIA & $19(31.7)$ \\
\hline Lymphadenectomy & $31(51.7)$ \\
\hline Sentinel lymph node dissection without & $9(15)$ \\
\hline lymphadenectomy & \\
\hline Cell differentation & $18(30)$ \\
\hline G2 & $32(53.3)$ \\
\hline G3 & $10(16.7)$ \\
\hline The dose of brachytherapy (Gy) & $3(5)$ \\
\hline 17 & $46(76.7)$ \\
\hline 18 & $1(1.7)$ \\
\hline 20 & $6(10)$ \\
\hline 21 & $1(1.7)$ \\
\hline 24 & $3(5)$ \\
\hline 27 & $27(45)$ \\
\hline Cylinder diameter (cm) & \\
\hline 2 & \\
\hline 3 & \\
\hline
\end{tabular}

lated with the formula for treatment of PDR, as reported elsewhere [4]. Calculated EQD2 at the tip point and at a $0.5 \mathrm{~cm}$ depth from the applicator surface are presented in Table 2. After treatment completion, all patients were followed-up regularly.

\section{Results}

After a median follow-up of 23 months, there were two (3\%) local treatment failures, in both cases including the lower vaginal metastases outside of the irradiated volume. One IC G1 patient relapsed 11 months after 27 Gy 2D BT. After exclusion of endometrial cancer spread by PET, she was successfully salvaged with surgery followed by pelvic irradiation at a dose of $50 \mathrm{~Gy} /$ 28 fractions with the concomitant boost of 4 Gy to the vaginal tumour. She has remained free of the disease for the subsequent three years. Another relapse was seen in an IC G2 case diagnosed 5 months after surgery and the 20 Gy BT, treated with palliative local irradiation and chemotherapy, who subsequently died due to cancer dissemination. Distant cancer metastases to the lung developed in one more patient.

Pulsed-dose-rate VBT was well tolerated. After therapy, 24 patients (40\%) complained of mild (grade 1 or 2) urinary, most frequently incontinence, and/or bowel complications. There was one case of postsurgical grade 3 urinary toxicity. No severe bowel effects were observed.

\section{Discussion}

In the PORTEC-2 trial after VBT, 5-year rates of locoregional (vaginal or pelvic recurrence, or both) and vaginal recurrence were $5.1 \%$ and $1.8 \%$, respectively at a median follow-up of 45 months (range: 18-78) [3]. In that trial, the reference isodose covered the proximal half of the vagina. The 5-year actuarial locoregional relapse rate of $5 \%$ and the vaginal recurrence rate of $2.7 \%$ in the VBT group alone were reported in another randomised trial, which compared external pelvic and vaginal irradiation versus vaginal irradiation alone in medium-risk group endometrial cancer patients [5]. In that trial, lowrisk and high-risk cases were included, thus the study populations were not fully comparable with those entered into the PORTEC-2. The interval time between surgery and VBT, with or without pelvic irradiation, was

Table 2. Calculated equivalent doses in 2 Gy fractions Type of applicator

EQD2 (Gy)

\begin{tabular}{|c|c|c|c|c|}
\hline \multirow{3}{*}{ 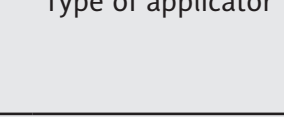 } & \\
\hline & \multicolumn{2}{|c|}{ At the tip point of cylinder } & \multicolumn{2}{|c|}{ At a $0.5 \mathrm{~cm}$ depth from applicator } \\
\hline & Entire group & 18 Gy subgroup & Entire group & 18 Gy subgroup \\
\hline \multicolumn{5}{|l|}{ Cylinder diameter $2 \mathrm{~cm}$} \\
\hline Range & $41.0-72.8$ & $41.0-61.4$ & $19.6-31.58$ & $20.7-20.8$ \\
\hline Mean/Median & $56.4 / 54.6$ & $52.2 / 50.7$ & $22.7 / 20.8$ & $21.8 / 20.8$ \\
\hline \multicolumn{5}{|l|}{ Cylinder diameter $3 \mathrm{~cm}^{\#}$} \\
\hline Range & - & $49.7-86.2$ & - & $20.7-20.8$ \\
\hline Mean/Median & - & 73.9/74.1 & - & $20.7 / 20.7$ \\
\hline
\end{tabular}

EQD2 - the equivalent dose in 2 Gy fraction, ${ }^{*}$ - all cases received 18 Gy 
4 to 8 weeks. The proximal two-thirds of the vaginal length was defined as the target volume, and the total doses delivered with HDR (as the main technique) or LDR were 17.7 Gy to 20 Gy in either six, three or one fraction (in LDR). Vagina relapse in seven cases (2\%), including one outside the irradiated volume, was reported in a large retrospective study of 382 intermediate-risk (also included high-risk cases) endometrial cancer patients treated with HDR VBT with a median dose of 21 Gy (range: 6-21) delivered in three fractions at 2-week intervals [6]. The target volume was one-half to two-thirds of the length of the vagina. In both series, the dose was specified at a depth of $0.5 \mathrm{~cm}$ from the surface of the vaginal cylinder. In our series of patients who met the PORTEC-2 trial inclusion criteria and were treated with PDR BT, two isolated vaginal relapses were observed, both outside volumes encompassed by 2D BT. One of the relapsed patients was salvaged with surgery and radiation.

Pelvic irradiation planning in a case of local relapse after the vaginal vault BT is complex and should avoid the overlap of treated areas when two separate planning systems are used, as it was in a case salvaged with radiotherapy. The risk of complications varies and is associated with the cumulative BT dose. The introduction of 3D treatment planning has provided more precise information on the doses received by adjacent critical structures from the post-operative intracavitary vaginal vault BT, and may facilitate radiotherapy planning in the case of local recurrence. A simple method that allows transition to 3D CT based vaginal cylinder BT planning was described [7]. Of note, for intravaginal BT, a multi-channel cylinder was shown to provide dose reduction to normal tissue as compared to the standard device with a single central channel [8].

Acute grade 1 and 2 gastrointestinal (EORTC-RTOG small/large intestine) toxic effects in $12.6 \%$ and a $<1 \%$ rate of grade 3 complications after VBT were reported in the PORTEC-2 [2]. In the abovementioned trial by Sorbe et al., all (grade 1 to 3 ) late reactions were recorded in $2.7 \%$ of cases administered VBT alone, with grade 3 urinary tract side effects in $0.8 \%$ [5]. Grade 3 or worse complications were reported in $0.8 \%$ of patients in the abovementioned retrospective study of HDR VBT to a lenght of $5 \mathrm{~cm}$ and $6 \mathrm{~cm}$ of the vagina [6]. The rate of late urinary reactions grade 1 and 2 of $7 \%$, and 3\%, respectively, was reported in another series utilizing HDR BT [9]. In that series, no severe side effects accompaning PDR VBT were noted.

The use of sole post-operative VBT in low to intermediate risk early stage patients with endometrial cancer has significantly increased in recent years but fractionation schedules and target volumes in the vaginal vault in this therapy need to be standardized. Apart from HRD and LDR techniques, which were used in the PORTEC-2 trial, with the dose prescribed to $0.5 \mathrm{~cm}$ depth as common fractionation schemes, there is a variety of fractionations in both techniques, which provide comparable results $[1,10]$. The American Brachytherapy Society guidelines formulated for adjuvant vaginal cuff BT (HDR and LDR) after a hysterectomy, recommends routine irradiation of the proximal $3-5 \mathrm{~cm}$ of the vagina [10].
Pulsed-dose-rate treatment is a relatively uncommon BT modality combining the physical advantage of HDR technology and radiobiological advantage of conventional continuous LDR BT. To achieve the equivalent of the LDR effect while using PDR treatment strategy, a similar total dose and an average dose rate should be considered. The recommended optimal dose rate with the PDR technique is from 0.4 to $0.7 \mathrm{~Gy} / \mathrm{h}$, however, the optimal timedose pattern for PDR is still debated. A dose of 1 Gy per pulse (i.e. the upper dose limit for keeping hypothetical favourable biological features of PDR) applied in endometrial cancer patients administered adjuvant VBT, is our intitutional policy. We have selected this dose for a practical reason - to shorten the treatment time. In a similar way to some other institutions, the PDR in our practice has replaced the LDR treatment.

The reported experience with the PDR technique in endometrial cancer is still limited. Four-dimentional image-guided adaptive PDR BT as a boost dose, following external beam radiotherapy, has been reported in locally recurrent endometrial cancer [11]. There is no reported study of sole PDR VBT in an adjuvant setting for endometrial cancer, which makes it impossible to compare this series to reports from other institutions.

The prescribed dose of 18 Gy in 18 hourly pulses was chosen following the calculation that it is equivalent to at least 45 Gy on the surface of the applicator [12]. In fact, with the use of the EQD2 formula as a recommended method of comparing the two fractionation schemes, this department's PDR treatment dose of 18 Gy is lower than the doses used in the PORTEC-2. For the tumor effect $(\alpha / \beta=10)$, the estimated LDR dose of 30 Gy reported in this trial with the use of EQD2 remains in a range of approximately 30.2 Gy to $32.2 \mathrm{~Gy}$, depending on a delivered dose rate of 0.5 to $0.7 \mathrm{~Gy} / \mathrm{h}$, respectively. In this series, the 18 Gy dose used in PDR is an equivalent dose of only $20 \mathrm{~Gy}$. In the Sorbe et al. trial [5] recalculated to $\operatorname{EQD} 2(\alpha / \beta=10)$, the total doses were 19.5 Gy to $23.5 \mathrm{~Gy}$ at a depth of $0.5 \mathrm{~cm}$ and $29.3 \mathrm{~Gy}$ to $35.3 \mathrm{~Gy}$ at the surface of the vaginal applicator.

It should be pointed out that the EQD2 dose at the tip point in our series is not the same across the applications, even in a subgroup treated with $18 \mathrm{~Gy}$. Both the choice of optimization algorithm, and optimization points profoundly affect the dose distribution in presented cases.

A much longer (median 74 days) time gap between surgery and the VBT procedure, as compared to that used in the PORTEC-2 trial (42.5 days), is another difference. Beside non-medical reasons, delaying the onset of VBT was necessary, since in many patients the cuff is not fully healed after a period of six weeks after hysterectomy. Indeed, vaginal dehiscence following HDR VBT, six weeks after laparoscopic robotic hysterectomies in two patients with endometrial carcinoma, was reported [13]. Hence, it is this department's policy to perform the VBT procedure at least two months after hysterectomy.

In the summary, post-operative PDR VBT at a dose of $18 \mathrm{~Gy}$ is safe and seems to provide adequate tumor control in high-intermediate risk endometrial cancer patients as defined in the PORTEC-2 trial. Nevertheless, close 
follow-up after VBT is warranted to implement a timely salvage therapy in cases with local relapses. Additional follow-up, on a larger number of patients is neccessary for final assessment of this therapy.

\section{Conclusions}

Post-operative PDR VBT at a dose of 18 Gy/18 pulses/every hour seems to be a safe technique and ensures vaginal control in high-intermediate risk endometrial cancer patients. Additional follow-up including a larger number of patients is necessary for the final assessment of this therapy.

\section{Disclosure}

Authors report no conflict of interest.

\section{References}

1. Kellas-Ślęczka S, Wojcieszek P, Białas B. Adjuvant vaginal brachytherapy as a part of management in early endometrial cancer. J Contemp Brachytherapy 2012; 4: 247-252.

2. Nout RA, Putter H, Jurgenliemk-Schultz IM et al. Quality of life after pelvic radiotherapy or vaginal brachytherapy for endometrial cancer: first results of the randomized PORTEC-2 trial. J Clin Oncol 2009; 27: 3547-3556.

3. Nout RA, Smit VT, Putter H et al. Vaginal brachytherapy versus pelvic external beam radiotherapy for patients with endometrial cancer of high-intermediate risk (PORTEC-2): an open-label, non-inferiority, randomized trial. Lancet 2010; 375: $816-823$

4. Couto JG, Bravo I, Pirraco R. Biological equivalence between LDR and PDR in cervical cancer: multifactor analysis using the linear-quadratic model. J Contemp Brachytherapy 2011; 3 : 134-141.

5. Sorbe B, Horvath G, Andersson H et al. External pelvic and vaginal irradiation versus vaginal irradiation alone as postoperative therapy in medium-risk endometrial carcinoma - a prospective randomised study. Int J Radiat Oncol Biol Phys 2012; 82: 1249-1255.

6. Alektiar KM, Venkatraman E, Chi DS et al. Intravaginal brachytherapy alone for intermediate-risk endometrial cancer. Int J Radiat Oncol Biol Phys 2005; 62: 111-117.

7. Malhotra $\mathrm{HK}, \mathrm{Wu} \mathrm{V}$, Whang $\mathrm{Z}$ et al. A novel method for vaginal cylinder treatment planning: a seamless transition to 3D brachytherapy. J Contemp Brachytherapy 2012; 4: 92-100.

8. Kuo HC, Mehta KJ, Yaparpalvi R et al. Feasibility study and optimum loading pattern of a multi-ring inflatable intravaginal applicator. J Contemp Brachytherapy 2013; 5: 93-100.

9. Donnelly ED, Rakhra S, Helenowski I et al. Dosimetry and toxicity outcomes in postoperative high-dose-rate intracavitary brachytherapy for endometrial carcinoma. J Contemp Brachytherapy 2012; 3: 135-140.

10. Small W Jr, Beriwal S, Demanes DJ et al. American Brachytherapy Society consensus guidelines for adjuvant vaginal cuff brachytherapy after hysterectomy. Brachytherapy 2012; 11: 58-67.

11. Fokdal L, Ørtoft G, Hansen ES et al. Toward four-dimentional image-quided brachytherapy in locally recurrent endometrial cancer. Brachytherapy 2014; 13: 554-561.

12. Serkies K, Blukis A, Tarnawska Z. Letter to the Editor. J Contemp Brachytherapy 2010; 2: 176.

13. Albuquerque K, Shah K, Potkul R. Vaginal cuff dehiscence after intracavitary brachytherapy for endometrial cancer. J Contemp Brachytherapy 2012; 4: 111-112. 